\title{
Real Life Application of Xenon: A Critical Review
}

\author{
Arvind Kumar Chhandak, Rekha Israni and A.V. Trivedi
}

Bhagwant University Sikar Road Ajmer, 305004, Rajasthan, India

*Corresponding author

\section{A B S T R A C T}

\section{Keywords}

Xenon, Real life application, Arc lamp,

High-intensity discharge Arc lamp, Application of xenon in anesthetics, High energy physics.

Article Info

Accepted:

21 July 2017

Available Online:

10 September 2017
This review was aimed to find out real life application of noble gas xenon. The noble gas xenon (Xe) occupy the zero group of the periodic table. It is fifth member of noble gas family. It is a colorless, dense, odorless noble gas found in the Earth's atmosphere in trace amounts. Xenon has so many application in real life, viz., in arc lamps in xenon HID (high-intensity discharge) lamp and in high pressure arc lamps to produce ultraviolet light. It is used in instruments for radiation detection, e.g., neutron and X-ray counters and bubble chambers. Xenon is used in medicine as a general anesthetic and in medical imaging. Modern ion thrusters for space travel use inert gases - especially xenon - for propellant, so there is no risk of the explosions associated with chemical propulsion. Xenon is also applied in high-energy physics for detecting nuclear radiation in bubble chambers. Furthermore, neuroscientists are experimenting with the use of xenon in diagnostic procedures to clarify x-ray images of the human brain.

\section{Introduction}

Xenon was discovered by Scottish chemist William Ramsay and English chemist Morris Travers in July 1898 at the University College London (Ramsay, Sir William, 2015). Xenon is a chemical element with symbol $\mathrm{Xe}$ and atomic number 54. Its atomic mass is 131.293 $\mathrm{u}$ (Meija, 2016) and electron configuration is [Kr] $4 \mathrm{~d}^{10} 5 \mathrm{~s}^{2} 5 \mathrm{p}^{6}$. It is a colorless, dense, odorless noble gas found in the Earth's atmosphere in trace amounts (Staff, 2007). Although generally unreactive, xenon can undergo a few chemical reactions such as the formation of xenon hexafluoroplatinate. The Earth's atmosphere contains about 0.1 part per million of xenon. It is also found as a component of gases emitted from some mineral springs. Xenon is obtained commercially as a by-product of the separation of air into oxygen and nitrogen.

\section{Real life application of xenon}

Xenon is used in photographic flashes, in high pressure arc lamps for motion picture projection and in high pressure arc lamps to produce ultraviolet light. It is used in instruments for radiation detection, e.g., neutron and X-ray counters and in bubble chambers. Xenon is used in medicine as a general anesthetic and in medical imaging. Modern ion thrusters for space travel use inert gases - especially xenon - for propellant, so there is no risk of the explosions associated with chemical propulsion. It is used in 
neuroscience in imaging and NMR, etc. Real life application of xenon are discussed below in detail:

\section{Application xenon in arc lamp}

A xenon arc lamp is a specialized type of gas discharge lamp, an electric light that produces light by passing electricity through ionized xenon gas at high pressure (Breeze, 1972) It produces a bright white light that closely mimics natural sunlight (Light Equipment, 2009). Xenon arc lamps can be roughly divided into three categories: continuousoutput xenon short-arc lamps, continuousoutput xenon long-arc lamps, and xenon flash lamps. Each consists of a fused quartz or other heat resistant glass arc tube, with a tungsten metal electrode at each end.

The glass tube is first evacuated and then refilled with xenon gas. For xenon flashtubes, a third "trigger" electrode usually surrounds the exterior of the arc tube. Xenon arc lamps are used in movie projectors in theaters in searchlights and for specialized uses in industry and research to simulate sunlight. It is used in flash photography.

Xenon is used in light-emitting devices called xenon flash lamps, used in stroboscopic lamps (Burke, 2003) to excite the active medium in lasers which then generate coherent light and, occasionally, in bactericidal lamps (Baltás et al., 2003). The first solid-state laser, invented in 1960, was pumped by a xenon flash lamp (Toyserkani et al., 2004) and lasers used to power inertial confinement fusion are also pumped by xenon flash lamps. First introduced in the 1940s, these lamps replaced the shorter-lived carbon arc lamps in movie projectors (Mellor, 2000). They are also employed in typical 35mm, IMAX, and digital film projection systems. They are an excellent source of short wavelength ultraviolet radiation and have intense emissions in the near infrared used in some night vision systems. Xenon is used as a starter gas in HID automotive headlights, and high-end "tactical" flashlights (Alchetron, 2017)

The individual cells in a plasma display contain a mixture of xenon and neon ionized with electrodes. The interaction of this plasma with the electrodes generates ultraviolet photons, which then excite the phosphor coating on the front of the display (Boeuf, 2003). Xenon is used as a "starter gas" in high pressure sodium lamps. It has the lowest thermal conductivity and lowest ionization potential of all the non-radioactive noble gases. As a noble gas, it does not interfere with the chemical reactions occurring in the operating lamp. The low thermal conductivity minimizes thermal losses in the lamp while in the operating state, and the low ionization potential causes the breakdown voltage of the gas to be relatively low in the cold state, which allows the lamp to be more easily started (Waymouth, 1979).

\section{Application of Pulse, dc and ac breakdown in high pressure gas discharge lamps}

An optical study of pulse, dc, and ac (50$400 \mathrm{kHz}$ ) ignition of metal halide xenon lamps has been performed by investigating intensified CCD camera images of the discharges. The ceramic lamp burners were filled with xenon gas at pressures of 300 and 700 mbar. In comparison with dc and pulse ignition, igniting with an $\mathrm{AC}$ voltage decreases the ignition voltage by up to $56 \%$ and the breakdown time scales get much longer $(\sim 10-3 \mathrm{~s}$ compared with $\sim 10-7 \mathrm{~s}$ for pulse ignition). Increasing the ac frequency decreases the ignition voltages and changes the ionization channel shapes. External irradiation of UV light can have either an increasing or a decreasing effect on ignition voltages (Beckers, 2008). 


\section{Application in lasers}

In 1962, a group of researchers at Bell Laboratories discovered laser action in xenon (Patel et al., 1962) and later found that the laser gain was improved by adding helium to the lasing medium (Bennett, 1962). The first excimer laser used a xenon dimer $\left(\mathrm{Xe}_{2}\right)$ energized by a beam of electrons to produce stimulated emission at an ultraviolet wavelength of $176 \mathrm{~nm}$ (Basov et al., 1971)

\section{Application of xenon in anesthesia}

Xenon has been used as a general anesthetic. Although it is expensive, because advances in recovery and recycling of xenon have made it economically viable (Tonner, 2006). Xenon interacts with many different receptors and ion channels, and like many theoretically multi-modal inhalation anesthetics, these interactions are likely complementary. Xenon is a high-affinity glycine-site NMDA receptor antagonist (Banks, 2010).

Like nitrous oxide and cyclopropane, xenon activates the two-pore domain potassium channel TREK-1. A related channel TASK-3 also implicated in the actions of inhalation anesthetics is insensitive to xenon (Gruss et al., 2004). Xenon inhibits $\mathrm{Ca}^{2+}$ ATPase by binding to a hydrophobic pore within the enzyme and preventing the enzyme from assuming active conformations (Lopez et al., 1995). Xenon has a minimum alveolar concentration (MAC) of $72 \%$ at age 40, making it $44 \%$ more potent than $\mathrm{N}_{2} \mathrm{O}$ as an anesthetic. Thus, it can be used with oxygen in concentrations that have a lower risk of hypoxia. Unlike nitrous oxide $\left(\mathrm{N}_{2} \mathrm{O}\right)$, xenon is not a greenhouse gas and is viewed as environmentally friendly (Goto et al., 2003) though recycled in modern systems, xenon vented to the atmosphere is only returning to its original source, without environmental impact.

\section{Application in neuroprotectant}

Xenon induces robust cardio protection and neuro protection through a variety of mechanisms. Through its influence on $\mathrm{Ca}^{2+}$, $\mathrm{K}^{+}$, KATP\HIF, and NMDA antagonism, xenon is neuroprotective when administered before, during and after ischemic insults (Schmidt et al., 2005). Xenon is a high affinity antagonist at the NMDA receptor glycine site (Banks et al., 2010).

Xenon mimics neuronal ischemic preconditioning by activating ATP sensitive potassium channels (Bantel et al., 2009). Xenon allosterically reduces ATP mediated channel activation inhibition independently of the sulfonylurea receptor-1 subunit, increasing KATP open-channel time and frequency.

Xenon gas was added to the ventilation mix for a newborn baby at St. Michael's Hospital, Bristol, England, whose life chances were otherwise grim, and was successful, leading to the authorisation of clinical trials for similar cases.

\section{Application in imaging}

Gamma emission from the radioisotope 133$\mathrm{Xe}$ of xenon can be used to image the heart, lungs, and brain, for example, by means of single photon emission computed tomography. 133-Xe has also been used to measure blood flow (Van Der Wall, 1992). Xenon, particularly hyperpolarized $129-\mathrm{Xe}$, is a useful contrast agent for magnetic resonance imaging (MRI). In the gas phase, it can image cavities in a porous sample, alveoli in lungs, or the flow of gases within the lungs (Albert and Balamore, 1998). Because xenon is soluble both in water and in hydrophobic solvents, it can image various soft living tissues (Wolber et al., 1998; Driehuys et al., 2009). 


\section{Application in NMR spectroscopy}

Because of the xenon atom's large, flexible outer electron shell, the NMR spectrum changes in response to surrounding conditions and can be used to monitor the surrounding chemical circumstances. For instance, xenon dissolved in water, xenon dissolved in hydrophobic solvent, and xenon associated with certain proteins can be distinguished by NMR (Luhmer, 1989). Hyperpolarized xenon can be used by surface chemists. Normally, it is difficult to characterize surfaces with NMR because signals from a surface are overwhelmed by signals from the atomic nuclei in the bulk of the sample, which are much more numerous than surface nuclei. However, nuclear spins on solid surfaces can be selectively polarized by transferring spin polarization to them from hyperpolarized xenon gas. This makes the surface signals strong enough to measure and distinguish from bulk signals (Raftery et al., 1997; Gaede et al., 1995).

\section{Other application of xenon}

In nuclear energy studies, xenon is used in bubble chambers (Galisonand et al., 1997) probes, and in other areas where a high molecular weight and inert chemistry is desirable. A by-product of nuclear weapon testing is the release of radioactive xenon-133 and xenon-135. These isotopes are monitored to ensure compliance with nuclear test ban treaties (Fontaine et al., 2004) and to confirm nuclear tests by states such as North Korea. A prototype of a xenon ion engine being tested at NASA's Jet Propulsion Laboratory. Liquid xenon is used in calorimeters (Gallucci, 2009) to measure gamma rays, and as a detector of hypothetical weakly interacting massive particles, or WIMPs. When a WIMP collides with a xenon nucleus, theory predicts it will impart enough energy to cause ionization and scintillation. Liquid xenon is useful for these experiments because its density makes dark matter interaction more likely and it permits a quiet detector through self-shielding.

Xenon is the preferred propellant for ion propulsion of spacecraft because it has low ionization potential per atomic weight and can be stored as a liquid at near room temperature (under high pressure), yet easily evaporated to feed the engine. Xenon is inert, environmentally friendly, and less corrosive to an ion engine than other fuels such as mercury or caesium. Xenon was first used for satellite ion engines during the 1970s (Zona, 2007). It was later employed as a propellant for JPL's Deep Space 1 probe, Europe's SMART-1 spacecraft and for the three ion propulsion engines on NASA's Dawn Spacecraft. Chemically, the perxenate compounds are used as oxidizing agents in analytical chemistry. Xenon difluoride is used as an etchant for silicon, particularly in the production of micro electromechanical systems (MEMS) (Brazzle et al., 1975). The anticancer drug 5-fluorouracil can be produced by reacting xenon difluoride with uracil (Staff, 2012). Xenon is also used in protein crystallography. Applied at pressures from 0.5 to $5 \mathrm{MPa}$ (5 to $50 \mathrm{~atm}$ ) to a protein crystal, xenon atoms bind in predominantly hydrophobic cavities, often creating a highquality, isomorphous, heavy-atom derivative that can be used for solving the phase problem (Drenth, 2007).

\section{Acknowledgement}

Authors thank all staff members of Bhagwant University Sikar Road Ajmer, 305004, Rajasthan, India for needful help and facilities.

\section{References}

Albert, M.S., and Balamore D. 1998. Development of hyperpolarized noble 
gas MRI. Nuclear Instruments and Methods in Physics Research A. 402 (2 - 3): 441 - 53.

Alchetron, 2017. https://alchetron.com/Xenon-5088598$\mathrm{W}$

Baltás, E. et al., 2003. A xenon-iodine electric discharge bactericidal lamp; Technical Physics Letters. 29 (10): 871 - 872.

Banks, P. et al., 2010. Competitive inhibition at the glycine site of the N-methyl-Daspartate receptor mediates xenon neuroprotection against hypoxiaischemia; Anesthesiology. 112 (3): 614 $-22$.

Bantel, C. et al., 2009. Neuronal preconditioning by inhalational anesthetics: evidence for the role of plasmalemmal adenosine triphosphatesensitive potassium channels. Anesthesiology. 110 (5): 986 - 95.

Basov, N.G. et al., 1971. Stimulated Emission in the Vacuum Ultraviolet Region. Soviet Journal of Quantum Electronics. 1 (1): 18 - 22.

Beckers, J., 2008. Pulse, dc and ac breakdown in high pressure gas discharge lamps. Journal of Physics D: Applied Physics. $41,14$.

Bennett, Jr., W. R., 1962. Gaseous optical masers; Applied Optics Supplement. 1: $24-61$.

Boeuf, J. P., 2003. Plasma display panels: physics, recent developments and key issues. J. Phys. D: Appl. Phys. 36.

Brazzle, J. D. et al., 1975. Modeling and Characterization of Sacrificial Polysilicon Etching Using Vapor-Phase Xenon Difluoride. Proceedings 17th IEEE International Conference on Micro Electro Mechanical Systems (MEMS). Maastricht, Netherlands: IEEE. pp. 737 - 740.

Breeze, R.H., and Ke B. 1972. Some Comments on Xenon Arc Lamp Stability. Review of Scientific
Instruments. 43, 821.

Burke, James. 2003. Twin Tracks: The Unexpected Origins of the Modern World. University Press. Oxford. p. 33.

Drenth, Jan; Mesters, Jeroen. 2007. The Solution of the Phase Problem by the Isomorphous Replacement Method. Principles of Protein X-Ray Crystallography (3rd Ed.) Springer New York pp. 123 - 171.

Driehuys, B. et al., 2009. Pulmonary perfusion and xenon gas exchange in rats: MR imaging with intravenous injection of hyperpolarized 129Xe. Radiology. 252 (2): 386 - 93.

Fontaine, J.P. et al., 2004. Atmospheric xenon radioactive isotope monitoring. Journal of Environmental Radioactivity. 72 (1 2): $129-35$.

Gaede, H. C. et al., 1995. High-field cross polarization NMR from laser-polarized xenon to surface nuclei. Applied Magnetic Resonance. 8, 3 - 4.

Galisonand Peter Louis et al., 1997. A Material Culture of Microphysics; University of Chicago Press. p. 339.

Gallucci, G., 2009. The MEG liquid xenon calorimeter. Journal of Physics: Conference Series. (1) 161.

Goto, T. et al., 2003. Will xenon be a stranger or a friend? The cost, benefit, and future of xenon anesthesia. Anesthesiology. 98 (1): 1 - 2 .

Gruss, M. et al., 2004. Two-pore-domain K+ channels are a novel target for the anesthetic gases xenon, nitrous oxide, and cyclopropane. Mol Pharmacol. 65 (2): 443 - 52

Light Equipment. 2009. http://www.lightingequipment.com/light-sources/gasdischarge-lamp/

Lopez, Maria M. et al., 1995. How Do Volatile Anesthetics Inhibit $\mathrm{Ca} 2+-$ ATPases? The Journal of Biological Chemistry. 270 (47): 28239 - 28245.

Luhmer, M., 1989. Interpretation of the 
solvent effect on the screening constant of Xe-129. Magnetic Resonance in Chemistry. 27 (10): 950 - 952.

Meija, J. et al., 2016. Atomic weights of the elements 2013 (IUPAC Technical Report). Pure Appl. Chem. 88 (3): 265 91.

Mellor, David. 2000. Sound Person's Guide to Video. Focal Press. p. 186

Patel, C. K. N. et al., 1962. Infrared spectroscopy using stimulated emission techniques; Physical Review Letters. 9 (3): 102 - 104.

Raftery, Daniel et al., 1997. Optical Pumping and Magic Angle Spinning: Sensitivity and Resolution Enhancement for Surface NMR Obtained with LaserPolarized Xenon. Journal of the American Chemical Society. 119 (37): 8746 - 8747.

Schmidt, Michael et al., 2005. Xenon Attenuates Cerebral Damage after Ischemia in Pigs. Anesthesiology. 102 (5): 929 - 936.

Sir William, Ramsay. 2015. Nobel Lecture The Rare Gases of the Atmosphere (December 12, 1904). nobelprize.org.
Staff, 2007. Xenon. Columbia Electronic Encyclopedia, (6th Ed.) Columbia University Press, Columbia.

Staff, 2012 Neil Bartlett and the Reactive Noble Gases; American Chemical Society.

Tonner, P. H., 2006. Xenon: one small step for anaesthesia? (Editorial review). Current Opinion in Anaesthesiology; 19 (4): 382 - 4

Toyserkani, E. et al., 2004. Laser Cladding. CRC Press; p. 48.

Van Der Wall, Ernst. 1992. What's New in Cardiac Imaging? SPECT, PET, and MRI. Springer.

Waymouth, John. 1979. Electric Discharge Lamps. Cambridge, MA: MIT Press.

Wolber, J. et al., 1998. Intravascular delivery of hyperpolarized 129Xenon for in vivo MRI. Applied Magnetic Resonance. 15 (3-4): 343 - 352.

Zona, Kathleen. 2007. Innovative Engines: Glenn Ion Propulsion Research Tames the Challenges of 21 st century Space Travel. NASA.

\section{How to cite this article:}

Arvind Kumar Chhandak, Rekha Israni and Trivedi, A.V. 2017. Real Life Application of Xenon: A Critical Review. Int.J.Curr.Microbiol.App.Sci. 6(9): 2063-2068.

doi: https://doi.org/10.20546/ijcmas.2017.609.253 